\title{
Processo de Abstração de Erros nas Análises Funcionais de Programas Aplicativos Fiscais
}

\author{
Everaldo Artur Grahl, Daniel Severo Estrázulas \\ Departamento de Sistemas e Computação \\ Universidade Regional de Blumenau (FURB) - Blumenau, SC - Brasil \\ egrahl@furb.br, pafdaniel@gmail.com
}

\begin{abstract}
Resumo. Neste artigo é relatada a experiência de aplicação do processo de abstração de erros nas análises funcionais de programas aplicativos fiscais. Estas análises são realizadas por órgãos técnicos credenciados, a partir de diversos testes funcionais derivados de requisitos legais exigidos pelo fisco. $O$ processo foi conduzido a partir dos registros de não conformidades de cento $e$ quarenta e uma avaliações realizadas por um órgão técnico credenciado. $O$ estudo realizado permitiu identificar seis grupos de erros no domínio de aplicativos fiscais incluindo falta total ou parcial de requisitos $e$ implementação incorreta destes requisitos.
\end{abstract}

\begin{abstract}
In this paper we report the experience of implementing the error abstraction process in functional analyzes of tax application programs. These analyzes are performed by agencies accredited technicians from various functional tests derived from legal requirements required by the tax authorities. The process was conducted based on the records of noncompliance of one hundred forty-one evaluations performed by an agency certified technician. The study identified six groups of errors in the field of tax applications including total or partial lack of requirements and incorrect implementation of this requirements.
\end{abstract}

\section{Introdução}

PAF-ECF é um Programa Aplicativo Fiscal que faz a interface com ECF (Emissor de Cupom Fiscal). O ECF é um equipamento de automação comercial com capacidade para emitir documentos fiscais e realizar controles de natureza fiscal, referentes a operações de circulação de mercadorias ou a prestações de serviços [Souza 2007]. Um bom histórico sobre o ECF pode ser visto em [Elias 2006].

Durante muito tempo no Brasil cada estado determinava as normas e regras a serem aplicadas sobre tal aplicativo. Com o objetivo de centralizar e extinguir essas diferentes normas e regras exigidas pelos diferentes estados sobre o Programa Aplicativo Fiscal, foi publicado em 2008 pelo fisco o Convênio ICMS 15/08 e o Ato COTEPE 06/08 de âmbito nacional. Esta legislação determina que todo Programa Aplicativo Fiscal deve passar por uma análise funcional em um órgão técnico credenciado para que o mesmo possa ser utilizado no varejo. De posse do laudo da análise funcional a empresa desenvolvedora deverá cadastrar a versão aprovada do programa aplicativo nos estados onde deseja atuar. Este conjunto de normas e leis estabelece regras e requisitos para os aplicativos comerciais. Durante as diversas 
avaliações realizadas pelo órgão técnico credenciado FURB identificaram-se problemas comuns e recorrentes. Decidiu-se por utilizar um processo que facilitasse a análise destes problemas.

O objetivo deste artigo é relatar a experiência de aplicação do processo de abstração de erros na análise de programas aplicativos fiscais permitindo identificar os principais problemas existentes. A abstração de erros consiste em analisar a natureza dos defeitos e determinar os erros responsáveis por tais defeitos.

O artigo é composto por quatro seções. Nesta primeira seção é contextualizado o problema e definido o objetivo do artigo. Na segunda seção são apresentadas algumas informações sobre o processo de análise funcional incluindo requisitos e testes exigidos. $\mathrm{Na}$ terceira seção é apresentado o resultado da aplicação da abstração de erros nas avaliações realizadas. Por último, são apresentadas as considerações finais com os principais resultados e conclusões, além de sugestões para trabalhos futuros.

\section{Processo de Análise Funcional}

Quando uma empresa de software submete o seu aplicativo para análise funcional num órgão técnico credenciado, deve atender várias funcionalidades exigidas pela legislação. Estas funcionalidades envolvem desde formato de arquivos, geração de relatórios fiscais até aspectos de segurança, portabilidade e confiabilidade da aplicação.

Atualmente um aplicativo fiscal deve atender pelo menos trinta e um requisitos de forma geral e dependendo do segmento que atua (posto de combustíveis, restaurante, oficina de conserto), terá que atender mais alguns requisitos. Estes requisitos, por sua vez, são analisados a partir de vários testes pertencentes a um roteiro de testes que o órgão credenciado deve adotar para sua análise. Este roteiro de testes é público e pode ser obtido no site do Conselho de Política Fazendária - [Confaz 2012]. Os tipos de testes utilizados são os testes de requisitos que tem o objetivo de verificar se o sistema executa corretamente as funcionalidades e se está em conformidade com as políticas e os procedimentos da organização. Estes testes geralmente são derivados diretamente dos requisitos e são realizados basicamente através da criação de condições de testes e checklists de funcionalidades [Bastos 2007]. A partir desta análise é emitido um laudo que será disponibilizado para a empresa e para o fisco.

No Quadro 1 é apresentada uma descrição do processo de Análise Funcional com propósito e resultados esperados. Esta descrição foi proposta pelos autores para facilitar o entendimento do processo realizado e foi derivada do processo de auditoria previsto na Norma ISO/IEC 12207 [ABNT 2009].

\section{Quadro 1 - Processo de Análise Funcional}

\section{Processo de Análise Funcional}

\section{Propósito}

O propósito do Processo de Análise Funcional é determinar, independentemente, a conformidade do programa aplicativo fiscal selecionado com os requisitos previstos na legislação. 


\section{Resultados esperados}

Como resultado da implementação bem-sucedida do Processo de Análise Funcional tem-se:

a) é desenvolvida e implementada uma estratégia de análise funcional;

b) a conformidade do programa aplicativo fiscal com os requisitos exigidos na legislação vigente é determinada de acordo com a estratégia de análise funcional;

c) os procedimentos e testes mínimos previstos no Roteiro de Análise Funcional devem ser cumpridos;

d) problemas de não conformidade detectados durante uma análise funcional são identificados, registrados e comunicados para os responsáveis pela ação corretiva e resolução, e

e) laudo técnico da análise funcional é elaborado e disponibilizado para as partes interessadas.

A seguir é apresentado no Quadro 2 um exemplo de um requisito de uso geral a ser atendido (requisito VII - item 6) e o respectivo teste exigido. Neste caso trata-se de um requisito com um teste. Um requisito pode exigir vários testes.

\section{Quadro 2 - Exemplo de Requisito e Teste exigido}

\section{Requisito VII - item 6}

O PAF-ECF deve, salvo quando da execução de comando de impressão de documento, em todas as suas telas, conter uma caixa de comando ou tecla de função identificada "MENU FISCAL", sem recursos para restrição de acesso, contendo categorias com as seguintes identificações e funções, exceto se a função não for disponibilizada pelo software básico do $E C F$, hipótese em que deverá apresentar a mensagem "Função não suportada pelo modelo de ECF utilizado": "Arq. MFD" para gerar arquivo eletrônico da Memória de Fita Detalhe conforme leiaute estabelecido no Ato COTEPE/ICMS 17/04 com possibilidade de seleção por período de data e por intervalo de COO, no mesmo subdiretório onde está instalado o PAFECF ou $S G$, quando este executar esta função, devendo o programa aplicativo informar o local da gravação e assiná-lo digitalmente, inserindo ao final do arquivo uma linha com o registro tipo EAD.

\section{TESTE 024: Arquivo Eletrônico da MFD AC17/04 - Função “Arq. MFD” por COO}

Passo 1: Execute a função “Arq. MFD” do Menu Fiscal e selecione o intervalo de COO.

Passo 2: Observe se a função foi executada ou se foi apresentada a mensagem "Função não suportada pelo modelo de ECF utilizado".

Passo 3: Caso o arquivo tenha sido gerado, verifique se o mesmo foi gravado no subdiretório onde está instalado o PAF-ECF ou SG, quando este executar esta função, devendo o programa aplicativo informar o local da gravação e execute a validação da assinatura digital do arquivo 
(Registro tipo EAD) utilizando o aplicativo eECFc observando as instruções constantes no item XIX das Orientações Gerais deste Roteiro.

Condição para requisito atendido: Geração do arquivo TXT AC17/04 da MFD no subdiretório onde está instalado o PAF-ECF ou SG, quando este executar esta função, informando o local da gravação, o arquivo TXT deve conter o registro tipo EAD (Assinatura Digital) devidamente validado OU a apresentação da mensagem descrita no passo 2.

Condição para requisito não atendido: Inexistência de função para gerar o arquivo TXT AC 17/04 da MFD OU gravação em subdiretório diverso do especificado no item 6 OU não apresentação da mensagem informando o local da gravação do arquivo OU não execução da geração do arquivo sem apresentação da mensagem descrita no passo 2 OU geração do arquivo sem o registro tipo EAD OU falta de validação da assinatura digital do arquivo.

\section{Abstração de Erros}

No artigo de [Lanubile 1998] são apresentadas diretrizes para aplicação do processo de abstração de erros. Para este trabalho estas diretrizes foram adaptadas para avaliar os problemas identificados nos programas aplicativos fiscais. Optou-se pelo processo de abstração de erros porque se mostrou um processo prático e também porque existiam muitos registros de defeitos que poderiam ser analisados rapidamente. Basicamente o processo utilizado foi composto de três etapas principais:

a) O programa é inspecionado para identificar defeitos.

b) Identifique erros que levaram aos defeitos, classificando os defeitos em grupos mais abstratos.

c) Para cada erro, retorne ao programa e certifique-se de ter localizado todos os defeitos.

Importante ressaltar que existem vários trabalhos publicados que tratam de taxonomias para classificação de defeitos. Destaca-se um levantamento muito completo realizado na tese de [Kalinowski 2011]. Para a classificação proposta levou-se em consideração os atributos tipo e natureza previstos no padrão [IEEE Std 1044 2009] Padrão para Classificação de Anomalias de Software, que pode assumir os seguintes valores: dados, interface, lógica, descrição, sintaxe, normas, outros, incorreto, omitido e incluído sem ser necessário. Optou-se por não estender nenhuma taxonomia existente, mas a partir do estudo feito, criaram-se grupos de erros adotando a terminologia voltada ao domínio estudado.

Foram avaliados cento e quarenta e um aplicativos que foram submetidos a análise funcional em 2012 pelo Laboratório de Qualidade de Software da FURB Órgão Técnico Credenciado situado em Santa Catarina. Todos estes aplicativos são pertencentes ao segmento de automação comercial. As principais tecnologias e linguagens usadas para o desenvolvimento destes softwares analisados foram Delphi, VB, C\#, Visual Fox Pro e Java. Para cada avaliação foram registrados os problemas (não conformidades), num sistema web interno de suporte a avaliações. Estes registros foram a base para o processo de abstração de erros. A partir de consultas por palavras chaves a base de dados com os registros destas não conformidades, foi possível identificar alguns grupos de erros mais comuns. O resultado principal do processo de abstração adotado foi a criação da classificação dos grupos. Este processo de abstração 
foi realizado pelos autores que possuem formação em computação e experiência em testes de software. Os autores também foram responsáveis pelas avaliações dos programas aplicativos. Um exemplo de tela do sistema web que registrava as não conformidades pode ser visto na Figura 1.

1.23. TESTE 023: Arquivo Eletrônico da MFD AC17/04 - Função "Arq. MFD" por data

1.24. TESTE 024: Arquivo Eletrônico da MFD AC17/04 - Função "Arq. MFD" por COO

Resposta

$\mathrm{N}=$ Não Atende

Inexistência de função para gerar o arquivo TXT AC 17/04 da MFD

Observacao

1.25. TESTE 025: Arquivo Eletrônico da Tabela de Produtos

1.26. TESTE 026: Arquivo Eletrônico de Estoque

Figura 1 - Tela de Registro de Não Conformidade

Foram identificados seis grupos de erros com as seguintes ocorrências e respectivo percentual (Tabela 1) :

Tabela 1 - Ocorrências de Erros por Grupo

\begin{tabular}{|l|r|r|}
\hline \multicolumn{1}{|c|}{ Grupos de Erros } & \multicolumn{1}{c|}{ Ocorrências } & \multicolumn{1}{c|}{ \% } \\
\hline Arquivos do Menu Fiscal & 302 & $\mathbf{2 6 , 4}$ \\
\hline Funções não desenvolvidas & 12 & $\mathbf{1 , 1}$ \\
\hline Relatórios Gerenciais & 234 & $\mathbf{2 0 , 5}$ \\
\hline Funções com DLL de ECF & 54 & $\mathbf{4 , 7}$ \\
\hline Funções mal interpretadas & 110 & $\mathbf{9 , 6}$ \\
\hline Validações e inconsistências & 430 & $\mathbf{3 7 , 7}$ \\
\hline
\end{tabular}

Os seis grupos identificados são descritos a seguir, assim como são propostas recomendações para apoiar as organizações a eliminar os erros mais comuns : 


\section{Grupo 1 - Arquivos do Menu Fiscal}

Nesta categoria foram agrupados os problemas encontrados nas funções de geração de arquivos eletrônicos através do menu fiscal com base na especificação de requisitos do Fisco. Estes problemas geralmente são defeitos na formatação do layout do arquivo. Como exemplos de arquivos com mais problemas encontram-se: Vendas do período; Movimento por ECF e Documentos Auxiliares de Venda Emitidos. O principal erro deste grupo é a implementação incorreta dos requisitos exigidos para a formatação dos arquivos. Recomenda-se a realização de análises dos arquivos através de checagem automática como softwares validadores que podem agilizar o processo e apresentar resultados mais precisos.

\section{Grupo 2 - Funções não desenvolvidas}

Nesta categoria foram agrupados problemas relacionados a inexistência de funções exigidas pelo fisco. Muitos destes problemas são ocasionados pela falta de atenção da equipe de desenvolvimento, omitindo detalhes descritos nos requisitos. Como exemplos de problemas encontrados incluem-se: Função de arquivo de Memória Fita Detalhe não permite filtrar por contador de ordem de operação, somente por data; Função de mesclar pré-venda não existente e Falta da função de parâmetros de configuração do menu fiscal. Os erros deste grupo se referem a não implementação de funções que haviam sido especificadas nos requisitos. Recomenda-se um gerenciamento dos requisitos através de um processo de rastreabilidade que permitirá checar se todas as funções foram realmente implementadas.

\section{Grupo 3 - Relatórios Gerenciais}

Nesta categoria foram agrupados problemas sobre a incorreção nas informações impressas em relatórios gerencias. Os principais relatórios com problemas são: Identificação do PAF-ECF; Meios de Pagamento; Documentos Emitidos; Conferência de Mesas; Parâmetros de configuração e Orçamento por ECF. Os erros mais comuns nesta categoria são relacionados a interface (relatórios não contemplam informações exigidas). Recomenda-se uma boa leitura e interpretação dos requisitos exigidos para estes relatórios e a inclusão da prática de construção de protótipos de relatórios para validação dos mesmos.

\section{Grupo 4 - Funções com DLL de ECF}

Nesta categoria foram agrupados problemas relacionados a funções com DLL de ECF. Os erros em arquivos ocorrem em parte pelo código da empresa desenvolvedora e em parte pela função desenvolvida pelo fabricante da impressora de ECF. As funções que geraram mais erros foram: Arquivo da Memória de Fita Detalhe; Leitura da Memória Fiscal Completa pelo Ato Cotepe 17/04 e Espelho da Memória Fita Detalhe. O principal erro deste grupo é a implementação incorreta do requisito exigido, ocasionado muitas vezes por problema de lógica. Recomenda-se no caso de erros na biblioteca (DLL) fornecida pelo fabricante do ECF, a procura de DLLs mais atualizadas. Outra recomendação é implantar testes unitários que podem amenizar problemas como inconsistências na passagem de parâmetros destas DLLs. 


\section{Grupo 5 - Funções mal interpretadas}

Nesta categoria foram agrupados problemas relacionados a funções mal interpretadas. Alguns requisitos exigidos pelo fisco tem uma complexidade maior. $\mathrm{O}$ fisco admite em alguns casos a flexibilidade de funcionalidades, porém boa parte dos requisitos deve ser atendida, mesmo que não sejam aplicáveis ao segmento do aplicativo. Exemplos de funções complexas que geralmente são mal interpretadas: Mesclagem de duas ou mais pré-vendas; Mesclagem de dois ou mais documentos auxiliares de venda; Tabela de índices técnicos de produção e Controle de rastreamento de alterações no banco por usuários. O principal erro deste grupo é a implementação incorreta do requisito exigido, muitas vezes ocasionado por problema de lógica. Recomenda-se implantar testes unitários e uma boa leitura e interpretação dos requisitos exigidos.

\section{Grupo 6 - Validações e inconsistências}

Neste último grupo estão os erros mais genéricos não classificados nos outros grupos e que não são dependentes do domínio da aplicação. Como exemplos incluem-se: Falta de tratamento e validação na entrada de dados; Passagem de filtros inválida; Utilização de descontos e acréscimos negativos ou inválidos; Inconsistência com uso de campo inexistente no banco de dados e Problemas de sintaxe em consultas SQL. Recomenda-se aqui a utilização de testes de interface, testes de tratamento de erros e testes de regressão para evitar que manutenções resultem em novos defeitos.

\section{Considerações Finais}

As avaliações realizadas sobre os aplicativos fiscais demonstraram que as empresas ainda estão se adequando à legislação. Percebeu-se através de conversas informais com os desenvolvedores participantes das avaliações que existem muitas dúvidas sobre a interpretação dos requisitos exigidos. Apesar dos problemas identificados, a maioria dos requisitos foi atendida pelos aplicativos.

Neste artigo está sendo divulgado o processo de avaliação que está ocorrendo neste segmento atualmente no país e ainda indica os principais problemas identificados em avaliações realizadas. Estes problemas foram sistematizados e classificados através de um processo de abstração. O processo de abstração se mostrou prático e ajudou a identificar inconsistências na classificação. Isto foi percebido após a realização da terceira etapa do processo, que sugere uma revisão nos erros obtidos. Algumas classificações estavam equivocadas, principalmente relacionadas aos erros mais genéricos como validações e inconsistências.

Os grupos de problemas identificados poderão auxiliar as empresas em análises futuras no sentido de orienta-las sobre os principais motivos de reprovações ou não conformidades. Percebeu-se que 46,9\% dos erros identificados são relacionados à formatação dos arquivos e relatórios exigidos pela legislação. Ainda 37,7 \% dos erros são relacionados à falta de validações de entrada de dados e inconsistências na codificação. As recomendações apresentadas podem amenizar bastante a ocorrência destes problemas.

Para trabalhos futuros nesta área de análise funcional de programas aplicativos fiscais, sugere-se a concepção de um modelo de qualidade que contemple 
além da visão regulatória do fisco, a visão do desenvolvedor e do usuário. Desta forma, podem ser construídos programas aplicativos fiscais aderentes à legislação, mas também eficientes, seguros, confiáveis, fáceis de usar e mais completos para o mercado consumidor. Outro trabalho futuro poderia ser o estudo de padrões de requisitos legais para este domínio de aplicação, visto que os requisitos são muitas vezes mal interpretados e geram defeitos no software.

\section{REFERÊNCIAS}

ABNT (2009) - ASSOCIAÇÃO BRASILEIRA DE NORMAS TÉCNICAS. NBR ISO/IEC 12207 - Tecnologia de informação - Processos de ciclo de vida de software. Rio de Janeiro.

Bastos, Aderson. (2007) Base de conhecimento em teste de software. 2. ed. São Paulo : Martins Fontes.

CONFAZ (2012) - Roteiro de Análise Funcional de Programa Aplicativo Fiscal Emissor de Cupom Fiscal PAF-ECF. Brasília. Disponível em: http://www.fazenda.gov.br/confaz/confaz/_ Acesso em: 18 mar. 2013.

COTEPE 06/08. Ato COTEPE / ICMS 6, de 14 de abril de 2008. Dispõe sobre a especificação de requisitos do Programa Aplicativo Fiscal - Emissor de Cupom Fiscal (PAF-ECF) e do Sistema de Gestão utilizado por estabelecimento usuário de equipamento ECF e revoga o anexo I do Ato COTEPE 25/04. Brasília. Disponível em: http://www.fazenda.gov.br/confaz/confaz/atos/atos_cotepe/2008 Acesso em: 10 mar. 2013.

Elias, Paulo Roberto. (2006) "Utilização do sistema de informação do emissor de cupom fiscal como instrumento de gestão no setor supermercadista do Estado de Santa Catarina". 171 f, il. Dissertação (Mestrado em Ciências Contábeis) - Programa de Pós-Graduação em Ciências Contábeis, Centro de Ciências Sociais Aplicadas, Universidade Regional de Blumenau, Blumenau. Disponível em: $<$ http://www.bc.furb.br/docs/TE/2006/318820_1_1.pdf>. Acesso em: 18 mar. 2013.

ICMS 15/08. Convênio ICMS 15, de 04 de abril de 2008. Dispõe sobre normas e procedimentos relativos à análise de Programa Aplicativo Fiscal (PAF-ECF). Brasília. Disponível em http://www.fazenda.gov.br/confaz/confaz/Convenios/ Acesso em: 10 mar. 2013.

IEEE (2009). IEEE Standard Classification for Software Anomalies, Std 1044 - 2009

Kalinowski, Marcos. (2011) "Uma abordagem para prevenção de defeitos provenientes de inspeções para apoiar a melhoria dos processos de engenharia do software" - Rio de Janeiro: Tese de Doutorado UFRJ/COPPE.

Lanubile, F., Shull, F., and Basili, V.R. (1998) "Experimenting with error abstraction in requirements documents". In Proceedings of Fifth International Software Metrics Symposium, METRICS98. p. 114-121.

Souza, Silvania Mendes. (2007) - Guia Prático do emissor de cupom fiscal: aspectos operacionais e legais. São Paulo: IOB. 